\title{
The multiple effects of amino acids on the early stages of calcium carbonate crystallization
}

\author{
Andreas Picker, Matthias Kellermeier*, Jong Seto, Denis Gebauer* and Helmut Cölfen \\ Physical Chemistry, University of Konstanz, Universitätsstraße 10, Box 714, D-78457 Konstanz, Germany
}

Received August 10, 2012; accepted September 12, 2012

Published online: October 15, 2012

\section{Calcium carbonate / Amino acids / Nucleation / Crystal growth / Prenucleation clusters}

\begin{abstract}
Proteins have found their way into many of Nature's structures due to their structural stability, diversity in function and composition, and ability to be regulated as well as be regulators themselves. In this study, we investigate the constitutive amino acids that make up some of these proteins which are involved in $\mathrm{CaCO}_{3}$ mineralization - either in nucleation, crystal growth, or inhibition processes. By assaying all 20 amino acids with vapor diffusion and in situ potentiometric titration, we have found specific amino acids having multiple effects on the early stages of $\mathrm{CaCO}_{3}$ crystallization. These same amino acids have been independently implicated as constituents in liquid-like precursors that form mineralized tissues, processes believed to be key effects of biomineralization proteins in several biological model systems.
\end{abstract}

\section{Introduction}

The presence of an organic component in biogenic minerals, whether it is $0.2-40 \% \mathrm{w} / \mathrm{v}$, varies with the mineral system and the respective model organism being examined [1-6]. The organic matrix is chemically diverse, and often contains structural macromolecules like collagen or chitin [7-9], as well as proteins [10, 11], proteoglycans [12, 13] or polysaccharides [14]. All the different parts of the organic matrix are believed to play important roles in biomineralization processes. The composition of the biomineralization-related proteins are completely varied across species, however, many are found to have common characteristics such as highly glycosylated, acidic [15] and anionic functional groups [16-18]. The exact roles of these macromolecules are still unknown while what is known, is that this fraction is necessary for normal mineralized tissue growth and development, as well as its materials properties [19-22]. There are no consistent compositions or structural homologs found amongst these organic fractions, except

\footnotetext{
* Correspondence author

(e-mail: matthias.kellermeier@uni-konstanz.de,

denis.gebauer@uni-konstanz.de)
}

that they do have regions which are highly repetitive and have characteristics of "intrinsically disordered protein" (IDP) domains, domains implicated in either mineral binding or the assembly process [23-25]. Many have associated these IDPs to being active participants in various biological processes like biomineralization [24, 26-28].

Common sequence motifs found in these mineralization related proteins are often associated with long repeats of selected amino acids. In the sea urchin spicule, the SM50 matrix protein found in the larval spicule is found to have domains with repeats of glycine, proline and asparagine [29]. This differs with the repetitive domains found in mollusk shell extracellular proteins, which have long repeats of aspartic acid interspersed by a repeat block of valine, alanine, and glutamic acid [24]. It has been thought that these domains found in these proteins are themselves switches which are necessary for structural conformations that are responsible for specific $\mathrm{Ca}^{2+}$ mineral ion affinities [30]. Interestingly, these domains confer characteristics onto the protein that make it relatively insensitive to temperature induced aggregation, resistance to acid precipitation, and high apparent molecular masses. Many of these domains also are found to actively bind to $\mathrm{Ca}^{2+}$ ions themselves, not entirely excluding their possible direct roles in mineralization processes. This begs the question, whether structural motifs or the amino acid constituents themselves are major components affecting the mineralization process.

With the use of peptide synthesis methods, short peptides consisting of constitutive domains found in some of these just discussed mineralization related proteins can now be probed. Several groups have examined short peptides containing repeats of proline and glycine on mineralization [29, 31]. Others have used short peptides with consensus groups from specific known mineralization proteins [18, 26, 27, 32]. In many of these cases presented, the short peptides are often found to have specific affinity to a predominant crystalline face of the growing mineral $[22,33]$ or preference for a specific mineral polymorph [26, 27, 34]. In finding these short peptide sequences, groups have adapted molecular biology techniques to screen through the millions of sequences that have an affinity to a crystal growth step or face. One common technique includes the use of phage display, where a library of 
peptides is screened via affinity of virus head particles that express specific peptide sequences [22, 35, 36]. Using these techniques, we often find that the peptides themselves are short enough to be devoid of tertiary- and quaternary-structures, but continue to have dominant effects on crystal growth and nucleation. These results indicate that higher order structure may not be a necessary component in controlling or regulating mineralization, and suggest that the presence of specific amino acids alone may to some degree determine the influence on mineralization. Therefore, we have studied the influence of single amino acids on the crystallization of calcium carbonate.

In probing the effects of single amino acids on mineralization processes, especially the chirality of these amino acids on crystal morphology, several groups have used in situ techniques to observe crystal growth processes in solution. DeYoreo and coworkers have shown with in situ AFM that specific enantiomers of amino acids also have a significant effect on the growth steps of the crystal morphologies [37]. Tremel and coworkers have shown that specific enantiomers cause phase stabilization in calcium carbonate mineralization with micro droplets [38]. Work by Xie and coworkers closely examines effects of specific L-form amino acids on calcite crystal morphology [39]. Very recently, Pokroy et al. have revealed that all amino acids can get incorporated into a calcite host lattice causing distortions just like intracrystalline proteins [40]. Thereby, Asp and to a lesser extent Glu became incorporated into the lattice in relatively high concentrations, which was speculatively attributed to the longer side chain of Glu. Unexpectedly, Cys became incorporated in almost as high concentration as Asp which was explained by a potential thiol-calcium bond [40].

Here, we present an exhaustive study of the influence of all 20 amino acids found in Nature on the crystallization process of $\mathrm{CaCO}_{3}$ itself. Several - but by far not all - of them can influence calcium carbonate crystallization, whereas aspartic acid stands out in particular, showing the most pronounced effects throughout the early and also late stages of crystallization. We demonstrate that not only amino acid side chain chemistry, but also side chain configuration and amino acid stereochemistry play important roles. Our findings pose a first step towards a better understanding of the more complicated systems discussed above, where the amino acids are combined into peptides and proteins. All of this may help to ultimately obtain a better understanding of how proteins control $\mathrm{CaCO}_{3}$ mineralization processes in Nature.

\section{Experimental section}

\section{Materials}

The following chemicals were used as received: calcium chloride (Fluka, $1 \mathrm{M}$ volumetric solution), sodium hydroxide (Alfa Aesar, 0.01 M standard solution, or pellets from Roth, $\geq 98 \%$ ), hydrochloric acid (Merck, $1 \mathrm{M}$ standard solution), sodium carbonate (Aldrich, anhydrous, A.C.S grade), sodium bicarbonate (Riedel de Haën, A.C.S grade), sodium chloride (Riedel de-Han, A.C.S grade), ammonium carbonate (Acros Organics, analysis grade), Lalanine (Aldrich, 99\%), L-arginine (Acros, $\geq 98 \%$ ), L-asparagine (Aldrich, 99\%), L-aspartic acid (Aldrich, 98\%), D-aspartic acid (Aldrich, 99\%), DL-aspartic acid (Acros, $\geq 99 \%$ ), L-cysteine (Fluka, $\geq 99 \%$ ), L-glutamine (Sigma, $\geq 99 \%$ ), L-glutamic acid (Aldrich 99\%), D-glutamic acid (Aldrich 99\%), DL-glutamic acid monohydrate (Acros, 99\%), glycine (Aldrich, $\geq 99 \%$ ), L-histidine (Sigma, $\geq 99 \%$ ), L-isoleucine (Sigma, $\geq 98 \%$ ), L-leucine (Aldrich, $\geq 99.5 \%$ ), L-lysine (Sigma, $\geq 98 \%$ ), L-methionine (Sigma, 98\%), L-phenylalanine (Fluka, $\geq 99 \%$ ), L-proline (Aldrich, $\geq 99 \%$ ), L-serine (Fluka, $\geq 99.5 \%$ ), L-threonine (Sigma, 98\%), L-tryptophan (Aldrich, $\geq 99 \%$ ), L-tyrosine (Acros, 99\%), and L-valine (Aldrich, 99\%).

\section{Sample preparation}

In the titration experiments, stock solutions of $20 \mathrm{mM} \mathrm{L}$ amino acid and sodium chloride (20 and $40 \mathrm{mM}$ ) were prepared by dissolving appropriate amounts in Milli-Q water, while $0.01 \mathrm{M} \mathrm{NaOH}$ was used as solvent in the case of tyrosine, due to its poor solubility at near-neutral $\mathrm{pH}$. All amino acid stock solutions were then adjusted to pH 9.75 by adding aliquots of $1 \mathrm{M} \mathrm{HCl}$ and $4 \mathrm{M} \mathrm{NaOH}$, respectively. In a second step, $20 \mathrm{mM}$ carbonate buffer pH 9.75 was prepared by mixing suitable quantities of $20 \mathrm{mM} \mathrm{NaHCO} 3$ and $\mathrm{Na}_{2} \mathrm{CO}_{3}$. Subsequently, equal volumes of carbonate buffer and either water (reference), $\mathrm{NaCl}$ or amino acid stock solution were combined to yield samples containing $10 \mathrm{mM}$ both of carbonate and amino acid (or respectively 10 or $20 \mathrm{mM} \mathrm{NaCl}$ ). Furthermore, the effects of $\mathrm{pH}$, amino acid concentration and stereochemistry were also investigated using aspartate and glutamate as model systems. To that end, independent experiments were conducted in which (i) buffer and amino acid solutions were set to $\mathrm{pH} 9.25$ ( $\mathrm{pH}$ variation), (ii) stock solutions of 2 or $40 \mathrm{mM} \mathrm{L-Asp}$ and L-Glu were employed (concentration variation), and (iii) D-Asp, D-Glu, DL-Asp and DL-Glu were used instead of their optically pure L-forms under otherwise identical conditions (stereochemistry variation).

\section{In-situ potentiometric titration measurements}

Titration experiments were performed using a commercial, computer-controlled system from Metrohm (Filderstadt, Germany), operated with the custom-designed software Tiamo (v2.2). The setup consists of a titration device (Titrando 809) that regulates two dosing units (Dosino 807) capable of dispensing titrant solution in steps as small as $0.2 \mu \mathrm{L}$. The $\mathrm{pH}$ and free $\mathrm{Ca}^{2+}$ concentration in the samples during titration were monitored in real time utilizing a flat-membrane glass electrode (Metrohm, No. 6.0256.100) and a polymer-based ion-selective electrode (Metrohm, No. 6.0508.110), respectively.

All titrations were performed at room temperature with at least three repetitions per experiment (4-5 measurements were carried out for most samples). In a typical run, $25 \mathrm{ml}$ of the solution to be analyzed were filled into a $50 \mathrm{ml}$ beaker, and $10 \mathrm{mM} \mathrm{CaCl}_{2}$ (obtained by $1: 100$ dilution of a $1 \mathrm{M}$ standard) was added at a rate of $0.01 \mathrm{~mL} /$ 
min while stirring at $900 \mathrm{rpm}$. During $\mathrm{Ca}^{2+}$ addition, the $\mathrm{pH}$ was kept constant by automatic counter-titration of $0.01 \mathrm{M} \mathrm{NaOH}$, and the calcium potential was recorded. The ion-selective electrode was calibrated by dosing $10 \mathrm{mM} \mathrm{CaCl}_{2}$ into water (previously adjusted to the target $\mathrm{pH}$ by addition of $0.01 \mathrm{M} \mathrm{NaOH}$ ), using the same titration routine as in the actual measurements and applying a gentle stream of nitrogen on the solution so as to avoid extensive uptake of atmospheric $\mathrm{CO}_{2}$. Electrodes, beaker and burette tips were cleaned with $10 \%$ acetic acid and subsequently rinsed several times with distilled water after each experiment.

\section{$\mathrm{CaCO}_{3}$ crystallization by vapor diffusion}

Crystallization experiments according to the gas diffusion method [41] were carried out in a closed desiccator containing a petri dish with freshly powdered ammonium carbonate. The dish was covered with Parafilm, furnished with three pinholes utilizing a needle. Samples were prepared by combining $7.5 \mathrm{~mL}$ of a $20 \mathrm{mM} \mathrm{CaCl}_{2}$ solution with the same volume of $20 \mathrm{mM}$ amino acid stock (or water in case of the reference experiment). The mixtures were then filled into $20 \mathrm{~mL}$ glass vials, and small coverslips were placed at the bottom of the vials in order to facilitate crystal harvesting. Finally, the vials were sealed with Parafilm (likewise furnished with three pinholes) and exposed to the $\mathrm{CO}_{2} / \mathrm{NH}_{3}$ vapor from thermal decomposition of $\left(\mathrm{NH}_{4}\right)_{2} \mathrm{CO}_{3}$ in the desiccator. The initial $\mathrm{pH}$ of the solutions typically varied between 5.5 and 6.0 (due to the slight acidity of the $\mathrm{Ca}^{2+}$ ions). As tyrosine is not sufficiently soluble under these conditions, its concentration had to be decreased to a final value of $1 \mathrm{mM}$ in the experiments. Upon continued uptake of ammonia (and $\mathrm{CO}_{2}$ ), the $\mathrm{pH}$ of the samples rose to around 9.5, which presumably represents the $\mathrm{pH}$ where mineralization took place. Thus, the conditions under which crystallization occurred in these experiments are (at least roughly) comparable to those prevailing in the titration assays (pH 9.75). Eventually, after two weeks of exposure, the coverslips (now covered with crystals) were removed from the vials and rinsed with distilled water and acetone, followed by drying at $40^{\circ} \mathrm{C}$. Two weeks of ripening time were chosen to ensure equilibrium conditions as the gasdiffusion method was shown to be highly dependent on kinetic factors [42, 43]. Each amino acid was investigated twice in this manner, using different desiccators (though with identical inner volumes) in order to test reproducibility.

\section{Analytical methods}

Morphologies of the crystals grown by means of vapor diffusion were studied with a light microscope (Zeiss AXIO ImagerM2m), equipped with four different objectives $(10 \times / 0.2 \mathrm{pol}, 20 \times / 0.5 \mathrm{pol}, 50 \times / 0.8 \mathrm{pol}, 100 \times / 0.9 \mathrm{pol})$ and the corresponding polarizers with lambda filters. For scanning electron microscopy, the coverslips with the crystals were mounted onto stubs utilizing a double-sided adhesive carbon tape. Analyses were carried out using a tabletop system (Hitachi TM-3000), which was operated at $15 \mathrm{kV}$.

\section{Results and discussion}

In this work, we have studied the 20 natural proteinogenic L-amino acids concerning their influence on the early stages of calcium carbonate crystallization. For this purpose, a titration assay was employed that had previously been used to investigate the nucleation process [44] as well as the effect of distinct additives on precipitation scenarios [45-47]. As in earlier work, experiments were carried out by continually dosing $10 \mathrm{mM} \mathrm{CaCl}_{2}$ solution into an excess of $10 \mathrm{mM}$ sodium (bi)carbonate buffer, where the $\mathrm{pH}$ was kept constant at 9.75 by counter-titration of $10 \mathrm{mM} \mathrm{NaOH}$. From volumes of $\mathrm{CaCl}_{2}$ and $\mathrm{NaOH}$ added during the titration experiments, and the concentration of $\mathrm{Ca}^{2+}$ detected in solution by means of an ion-selective electrode, it is possible to derive the free and bound amounts of both $\mathrm{Ca}^{2+}$ ions and carbonate species at any given time. This allows for quantitative characterizations of solute clusters existing in the prenucleation regime and furthermore directly yields the solubility of the initially nucleated solid phase [44].

In a first series of experiments, we have screened all 20 amino acids at an initial concentration of $10 \mathrm{mM}$ in the carbonate buffer. L-amino acids were chosen due to their higher biological relevance as compared to D-enantiomers. In general, the reproducibility of the titration measurements was good, which is evident from fairly small error bars in most cases (see below). In the calcium curves discussed hereinafter, the amount of free calcium in the carbonate buffer at first increases linearly upon addition of $\mathrm{CaCl}_{2}$ solution at a constant rate; during this prenucleation stage, the detected concentration of free $\mathrm{Ca}^{2+}$ is always lower than what would be expected based on the amount of $\mathrm{CaCl}_{2}$ added, which is due to equimolar binding of

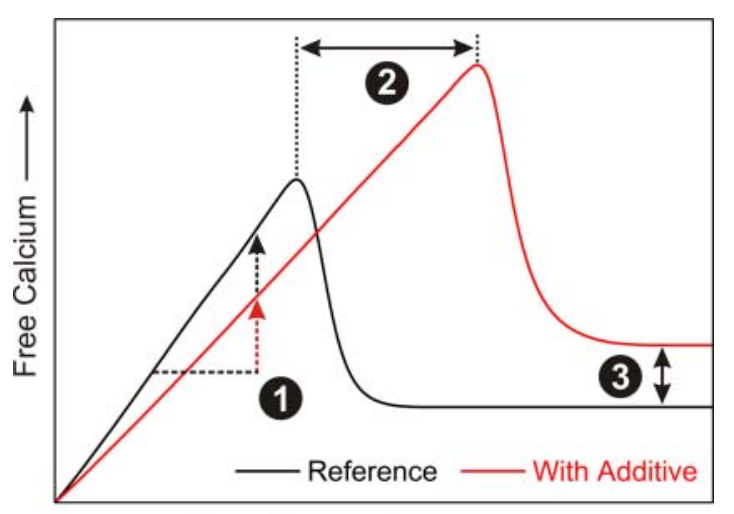

Time / Added Calcium $\longrightarrow$

Fig. 1. Qualitative development of the amount of free $\mathrm{Ca}^{2+}$ traced upon addition of dilute $\mathrm{CaCl}_{2}$ solution into carbonate buffer in the absence and presence of an additive. The criteria used to describe the influence of the investigated amino acids on the nucleation of calcium carbonate are; (1) the slope of the linear increase during the prenucleation stage (reflecting the fraction of $\mathrm{Ca}^{2+}$ bound in clusters and thus the apparent stability of prenucleation clusters); (2) the time of nucleation (showing any possible delaying effect of the additives); and (3) the level of free $\mathrm{Ca}^{2+}$ after nucleation (representing the solubility of the initially precipitated phase). Thus, the hypothetical additive depicted in the graph does (1) stabilize prenucleation clusters, (2) delay nucleation, and (3) favor an initial solid phase that is more soluble (and hence less stable) than what is obtained in the additivefree case. 
Fig. 2. Results of pH-constant titration assays showing the influence of different natural Lamino acids $(10 \mathrm{mM})$ on the nucleation of calcium carbonate, shown exemplarily for (a) alanine, (b) serine, (c) glutamic acid, and (d) aspartic acid. Three selected independent experiments are displayed in the graphs to illustrate reproducibility.
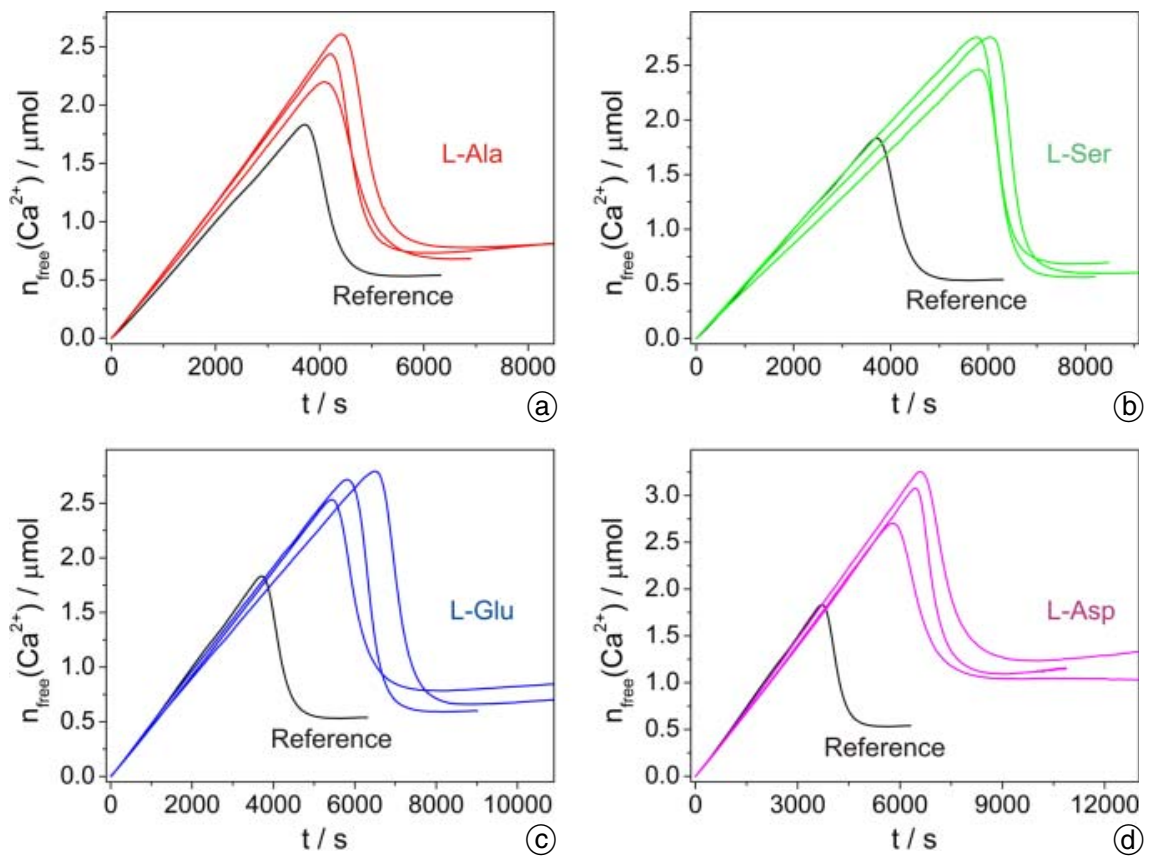

$\mathrm{Ca}^{2+}$ and $\mathrm{CO}_{3}{ }^{2-}$ ions in stable prenucleation clusters [44, 48]. When a critical point is reached, nucleation occurs and the amount of free calcium drops to a level that corresponds to the solubility of the precipitated $\mathrm{CaCO}_{3}$ phase and essentially remains unchanged upon further addition of calcium ions (due to the existence of a phase interface and hence a solubility product at this stage). In the present study, we focus on three major characteristics of the titration curves: (1) the slope of the calcium curves in the prenucleation regime (which reflects the stability of prenucleation clusters); (2) the time of nucleation (i.e. the peak maximum); and (3) the solubility product observed after nucleation (identifying the most soluble phase present at this stage). These circumstances are illustrated schematically in Fig. 1, where the possible modes of influence of the amino acids can be distinguished from a comparison of the titration curve recorded in the presence of an additive (red curve) to that found in its absence (reference scenario, black curve).

In Fig. 2, results obtained from the titration assays are shown exemplarily for four amino acids in terms of the development of the amount of detected free $\mathrm{Ca}^{2+}$ as a function of time during the early stages of precipitation. From these data, values for the prenucleation slope (in mol s${ }^{-1}$ ), the time of nucleation (in seconds), and the solubility of the nucleated phase (being defined as the product of the actual concentrations of free $\mathrm{Ca}^{2+}$ and $\mathrm{CO}_{3}{ }^{2-}$ ions, thus in $\mathrm{M}^{2}$ ) were determined by linear fits of the prenucleation regime, from the peak maximum, and by averaging ion products measured after nucleation, respectively. The values discussed in the following are the arithmetic mean calculated for each of the parameters from at least three independent measurements, with corresponding error bars depicting their standard deviation.

In order to be able to compare the data collected for the different amino acids, certain reference experiments have to be performed. As a primary reference, the evolution of free $\mathrm{Ca}^{2+}$ in neat carbonate buffer without any additives can be used [44]. However, since the chosen con- centration of amino acids is relatively high (equal to that of the buffer) and all of the investigated amino acids are charged at the given $\mathrm{pH}$ (due to fully deprotonated carboxylate groups, i.e. two negative charges for Asp and Glu, one negative charge for all other amino acids), the change in ionic strength caused by the additives is significant and related effects on $\mathrm{CaCO}_{3}$ precipitation must be taken into account. Hence, as a secondary reference, $\mathrm{Ca}^{2+}$ curves obtained in the presence of excess salt to resemble the additional salinity introduced by the amino acids, are to be considered; consequently, we have conducted measurements with carbonate buffers in which different amounts of sodium chloride had been dissolved $(20 \mathrm{mM}$ as a reference for Asp and Glu, $10 \mathrm{mM}$ for all other amino acids). Finally, the titration curve of glycine can be regarded as a third reference, as its "side chain" consists of a single hydrogen atom only. Thus, effects observed for this simple amino acid should largely be based upon the alpha- and gamma-functions, which are present in all amino acids; in turn, any distinct behavior found for other amino acids could then be ascribed to changes in the side chain.

Using these reference data for comparison, it is possible to discern effects that are caused by the chemistry and/ or structure of the amino acid, rather than simply its contribution to the overall ionic strength, and furthermore to potentially probe the role of side chain functional groups and configuration in the interaction with pre- and postnucleation calcium carbonate.

\section{Prenucleation Slopes}

The slope of the increase of free calcium prior to nucleation bears important information as it directly reflects interactions between additives and prenucleation species [45, 46]. Generally, a steeper slope implies that less calcium is bound in prenucleation clusters, indicating that they are destabilized by the particular additive, whereas flattening of the prenucleation regime in turn suggests cluster stabilization, as reported e.g. for citrate [46]. However, there are 


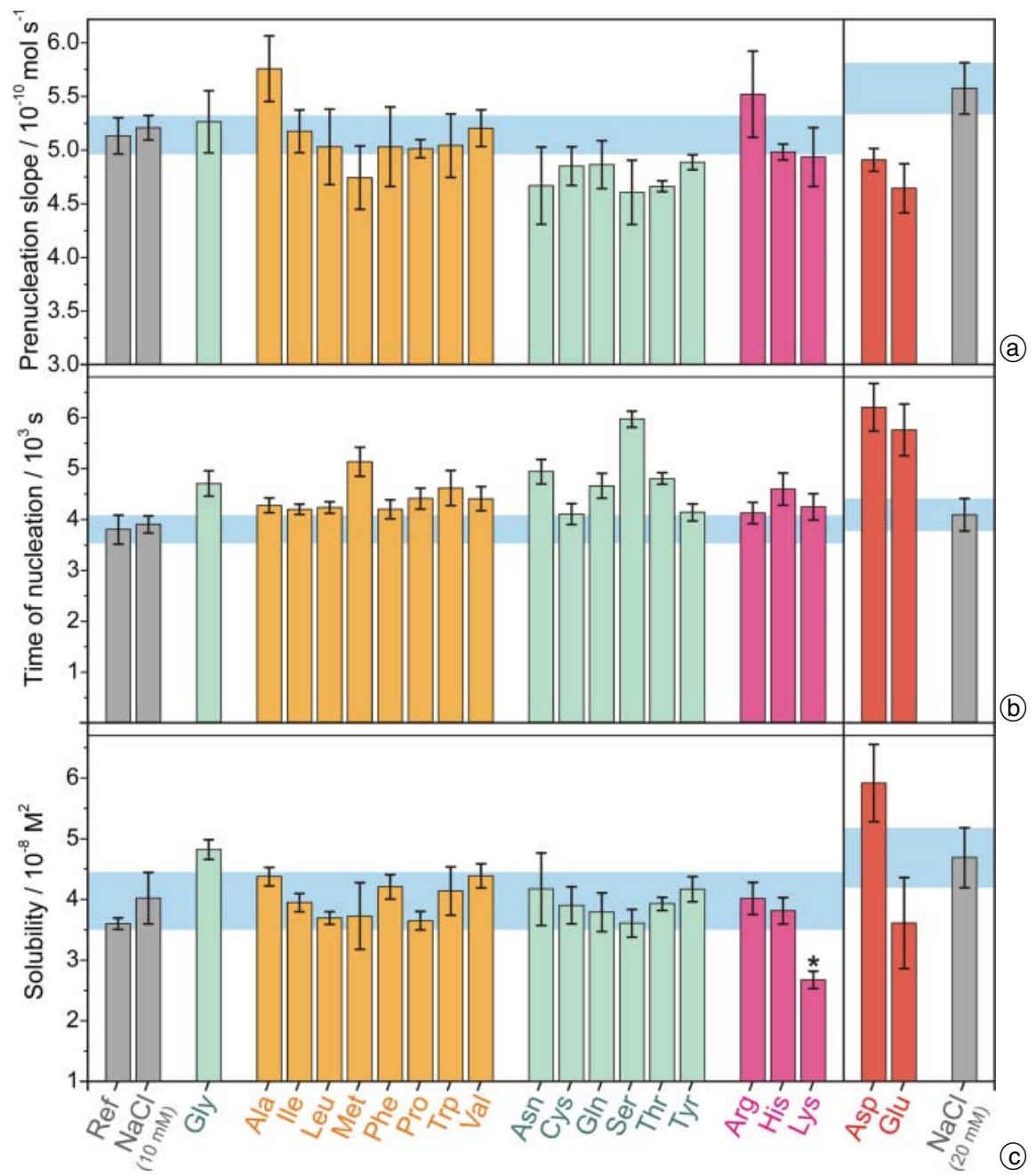

Fig. 3. Bar plots illustrating the effect of the 20 natural $\mathrm{L}$-amino acids $(10 \mathrm{mM})$ on $\mathrm{CaCO}_{3}$ nucleation at $\mathrm{pH} 9.75$ in terms of (a) the slope of the temporal increase in free $\mathrm{Ca}^{2+}$ prior to nucleation, (b) the time of nucleation, and (c) the solubility of the initially precipitated phase, all shown with error bars representing \pm one standard deviations (color code for the distinct groups of amino acids, green: polar/neutral, orange: non-polar/hydrophobic, magenta: basic, red: acidic). For comparison, values determined from the reference experiment without additives as well as from measurements in the presence of 10 and $20 \mathrm{mM} \mathrm{NaCl}$ are also shown (colored in grey). Note that $20 \mathrm{mM} \mathrm{NaCl}$ represents the increase in ionic strength caused by addition of Asp and Glu, while $10 \mathrm{mM} \mathrm{NaCl}$ resembles the salinity introduced by all other amino acids. Changes in the different parameters are judged to be significant if the corresponding value (and its standard deviation) lies outside the limits of error spanned by the additive-free reference and the $10 \mathrm{mM} \mathrm{NaCl}$ sample (for those amino acids carrying only one carboxylate group, blue-shaded interval in the left part of the graph), or respectively the $20 \mathrm{mM} \mathrm{NaCl}$ reference (for Asp and Glu, blue-shaded interval in the right part of the graph). ${ }^{*}$ The value for the solubility of the phase precipitated in the presence of lysine is not considered reliable, due to experimental errors caused by interference of that particular amino acid with the membrane of the Ca-selective electrode.

caveats as a flatter pre-nucleation slope can also result from activity effects due to increased ionic strength at high additive contents, as reported recently [49]. This would reduce the apparent amount of free $\mathrm{Ca}^{2+}$, given that the ion-selective measures activities rather than concentrations (i.e. the assumption of practically ideal solutions is no longer valid). Therefore, if the additive concentration is not low enough to become negligible with respect to changes in the salinity of the solutions (as is the case for the amino acids in this work), the calcium profiles have to be compared to reference measurements at equal ionic strength, as already mentioned above.

Figure $3 \mathrm{a}$ illustrates the influence of the different amino acids on the average slope of the prenucleation regime by means of bar plots derived from titration curves as those shown in Fig. 2, together with corresponding values obtained for the neat carbonate buffer as well as the two $\mathrm{NaCl}$ references. The amino acids are grouped according to their side chain chemistry in terms of non-polar/hydrophobic, polar/neutral, acidic and basic residues. First, it is worth noting that when $\mathrm{NaCl}$ is added to the carbonate buffer, the prenucleation slope increases (by about $9 \%$ at $20 \mathrm{mM}$ relative to the neat buffer). In fact, this trend is reverse to what would be expected for classical activity effects as outlined above, and suggests that, under the given conditions, the equilibrium constant of cluster formation decreases with growing ionic strength. Interestingly, in a recent titration study carried out at $\mathrm{pH} 9.00$, we could not detect any such destabilizing influence of $\mathrm{NaCl}$ on prenucleation clusters and actually observed a decrease in the slope due to activity issues [49]. Although we cannot explain these findings with certainty, one may speculate that distinct proto-structures inherent in the clusters at pH 9.00 and $9.75[44,50]$ could account for differing dependencies of their formation constant on the bulk ionic strength.

Regarding the effect of the amino acids on the prenucleation slope, we find that most of them do not induce changes relative to the references that exceed the limits of experimental error ( $c f$. Fig. 3a). The only amino acid apparently destabilizing $\mathrm{CaCO}_{3}$ clusters is alanine (and perhaps arginine, although the error bar is too large in this case to draw safe conclusions). On the other hand, there are several amino acids that clearly stabilize the clusters. Among these, glutamate and aspartate are most prominent (reducing the slope by ca. 16 and 12\%, respectively, relative to $20 \mathrm{mM} \mathrm{NaCl}$ ), followed by serine, threonine (both around $11 \%$ relative to $10 \mathrm{mM} \mathrm{NaCl}$ ) and tyrosine (ca. $6 \%$ ). This indicates that the side chain functionality is important for the capability of amino acids to stabilize prenucleation clusters, with carboxylate (Glu, Asp) and hydroxyl groups (Ser, Thr, Tyr) obviously being most efficient in this context. Apart from that, our data suggest that also asparagine and glutamine as well as cysteine and methio- 
nine may likewise have a stabilizing influence, which would confirm the notion that side chain chemistry plays a pivotal role here (Asn and Gln both being terminated by an amide group, Cys and Met both carrying a mercapto functionality). However, in view of respective error bars, these results cannot be regarded reliable without more exhaustive measurements. Nonetheless, it is evident that the structural configuration of the amino acids seems to be crucial for cluster stabilization, rather than sheer electrolyte properties, given that added $\mathrm{NaCl}$ has an opposite effect.

The fact that aspartate tends to stabilize $\mathrm{CaCO}_{3}$ prenucleation clusters is in good agreement with previous experiments performed with the same additive at $\mathrm{pH} 9.00$ where a similar, though much less pronounced influence was observed [49]. Again, one may infer that $\mathrm{pH}$-dependent changes in the structure of the clusters cause this difference; that is, aspartate might interact preferably with high-pH clusters, which were shown to exhibit lower binding strength and possibly feature a vaterite-like protostructure $[44,50]$.

The mechanisms underlying the effect of amino acids on the stability of the clusters remain unclear, in particular with respect to the found destabilization by alanine (and arginine). In turn, there is some evidence in literature that may hint towards the mode of stabilization exerted by Glu, Asp, Ser, Thr, and Tyr. Indeed, computer simulations performed by Finney and Rodger suggested that Asp molecules can effectively bind onto $\mathrm{CaCO}_{3}$ prenucleation clusters, thereby slowing down the dynamics of cluster dissociation (and re-assembly) [51]. This might shift the cluster equilibrium with the free ions towards the bound state, and thus explain the experimentally observed stabilization.

\section{Time of nucleation}

Average times of nucleation determined from the reference titration curves as well as those recorded in the presence of the different amino acids are shown in Fig. 3b. As compared to the prenucleation slope, the effect of added $\mathrm{NaCl}$ is less pronounced here, although a slight tendency towards inhibition of nucleation at higher ionic strength can be observed, in line with previous studies [49]. It is further obvious that most of the investigated amino acids delay nucleation relative to their respective $\mathrm{NaCl}$ reference, however to quite distinct extents. Only Phe, Cys, Tyr, Arg and Lys do not alter the time of nucleation to a degree that would be beyond the limits of error. In gener$\mathrm{al}$, the reproducibility of the values is fairly good, especially when noting that nucleation as such is a stochastic process and hence the time of nucleation should intimately depend on parameters like the precise volume of the solution, stirring rate, or temperature.

Unlike the results obtained for the stability of prenucleation clusters, there is no clear correlation between the side chain functionality of the amino acids and their capability to delay nucleation. The latter can be quantified by introducing a retarding factor $F$, defined as the quotient of the average nucleation time in the presence of amino acid and that of the respective $\mathrm{NaCl}$ reference. Corresponding values show that the most efficient amino acids again are serine $(F=1.53)$, aspartate $(F=1.52)$, and glutamate $(F=1.41)$. Thus, carboxylate functions seem to be beneficial for impeding nucleation, which is reasonable when considering that most industrial scale inhibitors are based on polyacrylates or consist of pure poly(aspartic acid) [52]. Also, poly-L-aspartic acid, poly-L-glutamic acid and poly-L-lysine were reported to be nucleation inhibitors for $\mathrm{CaCO}_{3}$ with the degree of delay increasing according to pAsp $>$ pGlu $\gg$ pLys [53].

Indeed, the effect of polyaspartates with different molar masses (comprising 50 and 200 monomer units) has already been investigated by titration assays as those used in the present work [46]. Retarding factors determined for these polymeric additives were much higher than those found for the monomers here, thus emphasizing the importance of chain length for the performance of antiscalant polymers. The strong influence of serine on the nucleation time is quite surprising, given that it only carries one uncharged $\mathrm{OH}$ group in its side chain and, in particular, that the other hydroxyl-bearing amino acids exhibit a much lower (threonine, $F=1.23$ ) or even vanishing effect (tyrosine, $F=1.06$ ). This suggests strongly that the chemical functionality of the amino acids does not (alone) determine the efficiency of nucleation inhibition, and that structural aspects, i.e. the configuration of the different functional groups, appear to be important as well. Similar arguments can be put forward when comparing the relatively strong inhibitor methionine $(F=1.31)$ with cysteine $(F=1.05)$, even though the polarity of these mercapto-functionalized amino acids is quite distinct. Further, the fairly pronounced retarding factor observed for glycine (1.20) is difficult to rationalize based on chemistry alone. In turn, we find rather consistent trends for the amidebearing amino acids Asn and Gln ( $F=1.26$ and 1.19) and the basic amino acids, which merely affect the nucleation time to a minor, if any extent. Likewise, the group of hydrophobic amino acids does generally not show strong inhibition of nucleation $(F=1.07-1.13)$ - with the exception of methionine and in part tryptophan $(F=1.18)$.

Again, we can only speculate about possible mechanisms causing delay of nucleation in the presence of certain amino acids. One conceivable scenario is that binding of the additives to prenucleation clusters, as proposed for Asp [51], results in an excess of negative charges in the periphery of the clusters, which then, electrostatically, hinders their aggregation. Such effects have recently been observed in solutions containing prenucleation clusters and negatively charged silicate species [54]. This might account for the high efficiency of Asp and Glu (two carboxylate functions), but in turn cannot explain the likewise drastic influence of serine. Another feasible mode of interference with nucleation also relies on colloidal stabilization of prenucleation clusters, but involving supramolecular assemblies of amino acids rather than simple monomers. In fact, recent experimental work has evidenced that amino acids themselves form (non-covalent) clusters in aqueous solutions at concentrations equal to those used in the present study [55], and computer simulations independently confirmed this notion for the case of aspartate [49]. Thus, one might hypothesize that such lar- 
ger entities can more effectively prevent $\mathrm{CaCO}_{3}$ prenucleation species from aggregation (and hence nucleation), in a manner roughly similar to steric stabilization of nanoparticle suspensions by means of polymer additives [49]. However, given that clustering has been reported for all of the investigated amino acids without any apparent trends with respect to functional groups [55], an explanation for the different efficiencies in nucleation inhibition on that basis remains elusive. In the end, this issue may relate to the question why some polymers, held together by covalent bonds, do exhibit differing modes of influence even though they carry the same chemical functions (e.g. poly(acrylic acid) $v s$. poly(aspartic acid) $[45,46])$, while other related polymers may not exhibit any influence at all. It appears that geometrical and conformational aspects could be important also in supramolecular polymers (prenucleation clusters) of amino acids [49].

\section{Solubility products}

In general, solubility products of nucleated phases correlate with the structure of the formed particles which, in the present case, can be amorphous (ACC) or represent different crystalline polymorphs (usually either calcite or vaterite under the given conditions). This is because solubility values directly reflect thermodynamic stabilities, and thereby may vary depending on particle size (GibbsThomson effects become important at the nanoscale) or the inclusion of impurities. As a common rule of thumb, higher solubility corresponds to lower stability. However, it has to be taken into account that, for mixtures of different polymorphs, the measured solubility is always dominated by the most soluble phase present, regardless of its quantity; consequently, the coexistence of less soluble phases in the system may be concealed.

Solubility products determined for the phases initially precipitated under the influence of the investigated amino acids and in the reference experiments are shown in Fig. 3c. In all cases, the magnitude of the ion product measured after nucleation is similar to what has previously been reported for titrations in the absence of additives [44], that is, the phase nucleated in the experiments appears to be amorphous calcium carbonate (ACC). Depending on $\mathrm{pH}$, the ACC particles can exhibit different solubilities, likely due to distinct short-range structures (and hence slightly dissimilar stabilities), and it has been shown that proto-calcite $(\mathrm{pc}) \mathrm{ACC}\left(K_{\mathrm{sp}} \approx 3.1 \cdot 10^{-8} \mathrm{M}^{2}\right)$ typically occurs at $\mathrm{pH}$ values $\leq 9.5$, while proto-vaterite (pv) ACC $\left(K_{\mathrm{sp}} \approx 3.8 \cdot 10^{-8} \mathrm{M}^{2}\right)$ is observed at higher $\mathrm{pH}[44,50]$. The solubility found in the present work for the amorphous phase formed in neat carbonate buffer $\left(K_{\mathrm{sp}} \approx 3.6 \cdot 10^{-8} \mathrm{M}^{2}\right)$ is indeed fairly close to the reported value for pv-ACC, thus supporting the notion of $\mathrm{pH}-\mathrm{de}-$ pendent proto-structuring.

Raising the ionic strength obviously leads to an increase in the level of the free ion product after nucleation (by about 12 and $30 \%$ for 10 and $20 \mathrm{mM} \mathrm{NaCl}$, respectively). Again, this effect is contrary to what would be expected for plain $\mathrm{Ca}^{2+}$ activity effects (which would decrease the solubility measured by the ion-selective electrode). This suggests that the solubility of the ACC phase is higher in the presence of additional electrolyte; an analogous effect of increased ionic strength on solubilities has been reported for calcite [56, 57].

Compared to their respective $\mathrm{NaCl}$ references, most of the studied amino acids do not show any marked influence on the solubility of the nucleated particles, considering the given limits of error. The only two amino acids significantly affecting the free ion product are glycine and aspartate (increasing the solubility by ca. 20 and $26 \%$, respectively). Slight changes might also be induced by alanine, phenylalanine, and valine (all towards higher solubility), but these trends are not regarded reliable based on the existing data (note that the very low ion product observed in the presence of lysine is not meaningful, as it originates from unspecific interactions of this amino acid with the polymer membrane of the ion-selective electrode, shown by reference experiments in lysine solutions without any carbonate).

The fact that glycine, being the simplest of all amino acids, has the strongest effect on solubility among those molecules carrying one carboxylate function is rather unexpected at first glance. One possible explanation for this finding could be that, due to its small size, glycine is capable of incorporating into the structure of the ACC particles, thereby rendering them less stable and more soluble. This notion is supported by the recent finding that glycine can become incorporated into the lattice of calcite (the concentration of incorporated Gly being among the highest of all amino acids) [40]. In turn, incorporation into the structure of ACC may no longer be possible as soon as a more bulky side chain is present in the amino acid.

As for the prenucleation slope and time of nucleation, the influence of aspartate on the solubility of the initially precipitated phase is quite pronounced - in contrast to glutamate and serine, which both stabilized prenucleation clusters and inhibited nucleation to a similar extent as Asp, but apparently do not alter the solubility (if any, Glu would even have an opposite effect). This is of particular interest with respect to the structures of Asp and Glu, which merely differ by one $\mathrm{CH}_{2}$ group. These results further underline the importance of side chain configuration, in addition to chemical functionality. Stabilization of transient $\mathrm{CaCO}_{3}$ phases with higher solubility in the presence of Asp has already been observed in recent titration studies [49], which however were carried out at lower $\mathrm{pH}$ (9.00). Under those conditions, the lifetime of the less stable intermediate was shorter than at the higher $\mathrm{pH}$ used in this work, and the free ion product dropped to pc-ACC during the later stages of the experiments. This indicates that the interaction of Asp with early $\mathrm{CaCO}_{3}$ species is more distinct in the high-pH regime, where proto-vateritic forms are expected (which is in line with the observations made concerning the effect on the stability of prenucleation clusters, cf. Section 3.1). Furthermore, it is worth noting that the influence of aspartate monomers on the initially nucleated phase is to some degree reminiscent of its polymeric analogue, given that polyaspartate is known to stabilize a metastable liquid-like precursor under certain circumstances, commonly referred to as PILP [58-60]. Titration experiments performed with pAsp present demonstrated the prolonged occurrence of a highly soluble phase 
Table 1. Identified modes of interaction between pre- and early postnucleation calcium carbonate and distinct amino acids, ranked according to their respective degree of influence.

\begin{tabular}{ll}
\hline Type of influence & Concerned amino acids \\
\hline Change of cluster stability & $\begin{array}{l}\text { stabilizing: Glu }>\text { Asp }>\text { Ser }>\text { Thr } \\
\text { destabilizing: Ala }\end{array}$ \\
Delay of nucleation & $\begin{array}{l}\text { Ser } \approx \text { Asp }>\text { Glu }>\text { Met }>\text { Asn } \\
\text { Thr }>\text { Gly } \approx \text { Gln } \approx \text { Trp } \approx \text { His }\end{array}$ \\
& $>$ Pro $\approx$ Val $(>$ Ala, Leu, Ile $)$
\end{tabular}

with varying composition $\left(K_{\mathrm{sp}} \approx 2 \cdot 10^{-7} \mathrm{M}^{2}\right)$, likely corresponding to PILP [46]. Thus, even though monomeric Asp affects the nature of the nucleated phase in a qualitatively similar manner, the efficiency of its polymeric form is still by far greater. The atomistic basis underlying the interaction between Asp and early postnucleation calcium carbonate may involve adsorption or inclusion to nucleated particles or rely on the action of non-covalent amino acid clusters as well, but definite conclusions cannot be drawn at this point.

Taken together, our titration experiments have shown that certain amino acid monomers can very well have a distinct impact on the early stages of calcium carbonate crystallization. The most outstanding amino acid in this context clearly is aspartate, which was found to be active in all of the investigated stages. Moreover, glutamate and serine proved to be quite efficient in stabilizing prenucleation clusters and delaying nucleation. The whole range of effects considered significant is summarized in Table 1. We note that the identified modes of interaction do not correlate with the reported concentrations of intracrystalline amino acids in calcite lattices [40], with the exception of Asp which appears to be special among all amino acids in any respect. For example, Pokroy et al. have found cysteine to be incorporated in calcite at concentrations as high as aspartic acid although only at comparatively higher cysteine concentrations [40]. Furthermore, glutamic acid showed rather high lattice incorporations. Factors governing lattice incorporation and lattice strain were acidity/basicity, the relative $\mathrm{p} K_{\mathrm{a}}$ values of the carboxyl and amino terminal groups, the amino acid size, and their rigidity [40]. As can be seen in Table 1, these correlations are not found in the prenucleation and early postnucleation stage with the exception of Asp. Cysteine is not found to have any significant influence and glutamic acid stabilizes prenucleation clusters and delays nucleation. Therefore, the action of amino acids in solution is very different from the state included into a calcite crystal lattice.

\section{Aspartic vs. glutamic acid}

As both aspartate and glutamate showed a relatively strong influence on the prenucleation slope and time of nucleation, and since subtle structural differences between the two amino acids appear to essentially determine their capability to interact with nucleated particles, these additives were investigated in more detail. In particular, further experiments were conducted in which the $\mathrm{pH}$ of the buf- fer, the amount of amino acid added, and the chirality of the additives were varied.

\section{Variation of $\mathbf{p H}$}

All experiments discussed so far were performed at $\mathrm{pH}$ 9.75. We have focused our investigations on this $\mathrm{pH}$ value, as it represents the experimentally found minimum of the $\mathrm{pH}$-dependent stability of prenucleation clusters [44] and, thus, the influence of additives should be maximal under these conditions [45]. In order to elucidate the role of cluster stability when it comes to the interaction with Asp and Glu, additional measurements were carried out at a lower $\mathrm{pH}$ of 9.25. As already stated above, there is strong evidence that the varying stabilities observed for prenucleation clusters at distinct $\mathrm{pH}$ levels reflect different structures [61]. This notion is based on the findings that a slightly less soluble amorphous phase (pc-ACC) is precipitated from more stable prenucleation clusters and vice ver$s a[44,50]$, and that nucleation of the ACC nanoparticles appears to rely on aggregation of the clusters [44, 54, 62]. While less stable pv-ACC has been obtained at $\mathrm{pH} 9.75$ in the absence of additives ( $c f$. Section 3.3), we expect pcACC to form from more stable prenucleation clusters in carbonate buffers at $\mathrm{pH} 9.25$. Indeed, this is confirmed by the free ion products determined after nucleation in the reference experiments at lower $\mathrm{pH}\left(K_{\mathrm{sp}} \approx 2.9 \cdot 10^{-8} \mathrm{M}^{2}\right.$, as compared to the literature value of ca. $\left.3.1 \cdot 10^{-8} \mathrm{M}^{2}[44]\right)$.

Comparative bar plots depicting $\mathrm{pH}$-dependent changes in the influence of Asp and Glu on $\mathrm{CaCO}_{3}$ nucleation are shown in Fig. 4. Although none of the observed effects is strong enough to exceed the limits of error, possible trends shall be briefly discussed. Concerning the slope of the prenucleation regime, we find that lowering the $\mathrm{pH}$ to 9.25 causes an additional flattening of the curve for both amino acids. This might suggest that Asp and Glu interact more readily with proto-calcitic clusters, stabilizing them to a slightly higher degree than their proto-vateritic counterparts at higher $\mathrm{pH}$. However, experiments performed with Asp at pH 9.00 do not support this tendency and show only minor influence of the amino acid on the stability of

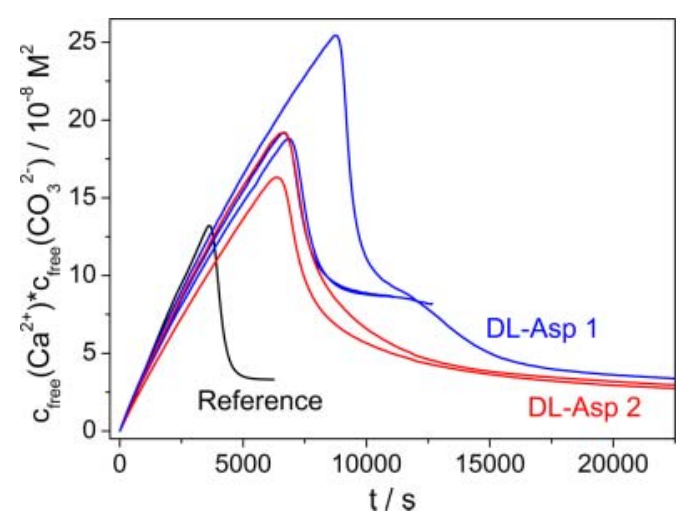

Fig. 4. Effect of $\mathrm{pH}$ on the interaction of L-Asp and L-Glu (both $10 \mathrm{mM}$ ) with pre- and postnucleation calcium carbonate, shown exemplarily for two distinct $\mathrm{pH}$ levels. Changes in the prenucleation slope (green), the nucleation time (red), and the solubility of the nucleated phase (blue) are given as factors on a relative scale with respect to the reference experiment in neat carbonate buffer (dashed grey line). 
prenucleation clusters under those conditions [49]. Further work will be required to ascertain whether the $\mathrm{pH}$ can significantly modulate interactions between prenucleation species and amino acids.

Regarding nucleation times, it seems as if inhibition by aspartate is stronger at lower than at higher $\mathrm{pH}$, whereas there is no noticeable change discernible in case of glutamate. In fact, retarding factors determined for Asp-containing solutions in the present and our previous study [49] give a consistent trend with $\mathrm{pH}$; relative to neat carbonate buffers at the corresponding $\mathrm{pH}$, we obtain the following values for $F$ : $1.63(\mathrm{pH} 9.75), 1.94(\mathrm{pH} 9.25)$, and 2.25 (pH 9.00). This confirms that the capability of Asp to delay nucleation indeed increases with decreasing $\mathrm{pH}$. Similar effects have been observed for oligomeric peptides, which exhibit distinct binding affinities to the different calcium carbonate polymorphs [47]. Again, a possible reasoning for this behavior could be based on the hypothesis that the amino acid interacts favorably with proto-calcite clusters prevailing at low $\mathrm{pH}$ and, thus, more efficiently hinders cluster aggregation (and, with it, nucleation).

Looking at the free ion products after nucleation, both Asp and Glu increase the solubility of the initially precipitated phases as compared to the neat carbonate buffer reference, and this effect appears to be more pronounced at lower $\mathrm{pH}$. However, solubility values on an absolute scale prove to be similar for Asp $\left(5.42 \cdot 10^{-8} \mathrm{M}^{2}\right.$ at $\mathrm{pH} 9.25 \mathrm{vs}$. $5.91 \cdot 10^{-8} \mathrm{M}^{2}$ at $\mathrm{pH} 9.75$, and about $4-5 \cdot 10^{-8} \mathrm{M}^{2}$ at $\mathrm{pH} 9.00$ [49] and almost identical in case of Glu (3.63 $10^{-8} \mathrm{M}^{2}$ at $\mathrm{pH} 9.25 \mathrm{vs.} 3.61 \cdot 10^{-8} \mathrm{M}^{2}$ at $\left.\mathrm{pH} 9.75\right)$. This indicates that the nature of the incipient ACC particles does not depend on $\mathrm{pH}$ and is essentially controlled by the additives. Interestingly, the solubility found in the presence of Glu at $\mathrm{pH} 9.25$ relates to that of pv-ACC, which usually occurs only at higher $\mathrm{pH}$ in the absence of additives. This suggests that glutamate favors nucleation of proto-vateritic phases over otherwise obtained proto-calcite ACC. In the case of aspartate, structural analyses of the nucleated particles would be required to identify the type of short-range order in the precipitated material, even though - as described above - there are indications for the formation of pc-ACC in the presence of this amino acid.

\section{Variation of the amino acid concentration}

In order to study the effect of additive concentration, further titrations were performed in which the content of Asp and Glu in the buffer was decreased to $1 \mathrm{mM}$ or increased to $20 \mathrm{mM}$ (all at the standard $\mathrm{pH}$ of 9.75). Corresponding results are shown in Fig. 5. At $1 \mathrm{mM}$, both amino acids do not influence $\mathrm{CaCO}_{3}$ nucleation to any significant extent (apart from, possibly, a slight increase in the solubility of the precipitated phase, which however might also be due to ionic strength-related effects). As outlined in the previous sections, Asp and Glu tend to stabilize prenucleation clusters and inhibit nucleation at $10 \mathrm{mM}$, while aspartate does in addition induce the formation of a more soluble initial phase. This behavior hints at the existence of a certain threshold in concentration, beyond which the amino acids start to become active.

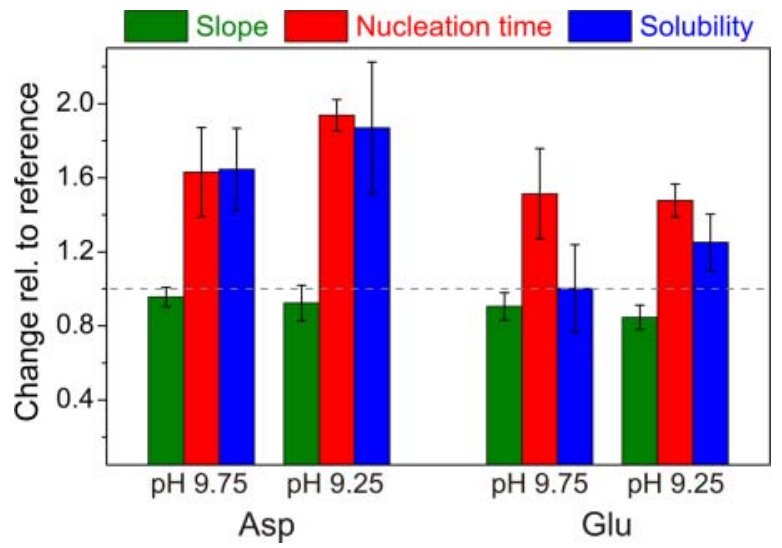

Fig. 5. Trends in the prenucleation slope, nucleation time, and solubility observed upon variation of the concentration of added L-Asp and L-Glu in experiments at $\mathrm{pH} 9.75$.

Increasing the amino acid content to $20 \mathrm{mM}$ leads to further flattening of the prenucleation regime as compared to the reference experiment in both cases. This is clear evidence that Asp and Glu are indeed capable of stabilizing prenucleation clusters (especially when considering that the ionic strength caused by addition of $20 \mathrm{mM}$ amino acid should have a (strong) opposite effect). In this context, the influence of glutamate seems to increase continuously with increasing concentration, whereas there is a quite abrupt change in the efficiency of aspartate between 10 and $20 \mathrm{mM}$. Nucleation times determined at high additive contents suggest that the inhibiting effect of Glu has virtually reached its maximum already at $10 \mathrm{mM}$, as no pronounced additional delay could be discerned at $20 \mathrm{mM}$. This is different in the case of Asp, where the retarding factor found at $20 \mathrm{mM}$ amino acid $(F=2.10)$ is still drastically higher than at $10 \mathrm{mM}(F=1.63)$. Concerning the free ion product after nucleation, quite the contrary is observed, as the solubilities measured in the presence of 10 and $20 \mathrm{mM}$ Asp are identical within error $\left(5.70 \cdot 10^{-8} \mathrm{M}^{2}\right.$ at $20 \mathrm{mM} v$ s. $5.91 \cdot 10^{-8} \mathrm{M}^{2}$ at $\left.10 \mathrm{mM}\right)$, while Glu becomes active in stabilizing a more soluble phase only at $20 \mathrm{mM}\left(K_{\mathrm{sp}}=4.72 \cdot 10^{-8} \mathrm{M}^{2}\right.$ at $20 \mathrm{mM} v s$. $3.61 \cdot 10^{-8} \mathrm{M}^{2}$ at $10 \mathrm{mM}$ ). These findings further emphasize that Asp and Glu exhibit distinct modes of interaction with pre- and postnucleation calcium carbonate, which cannot rely on chemical functionality alone but maybe also on the clustering of these two amino acids which was recently reported to be different [55].

\section{Variation of stereochemistry}

Recent studies have shown that polymorph selection of $\mathrm{CaCO}_{3}$ may depend on the chirality of amino acids present during crystallization [38]. By means of gas diffusion experiments, Wolf et al. found that L-alanine induces precipitation of vaterite, whereas its D-enantiomer gave predominantly calcite and the racemic mixture yielded exclusively calcite. In this regard, we have investigated the influence of amino acid chirality on the early stages of $\mathrm{CaCO}_{3}$ mineralization by means of titration measurements, using L- and D-enantiomers of both Asp and Glu as well as their racemic mixtures as additives (all at a concentra- 


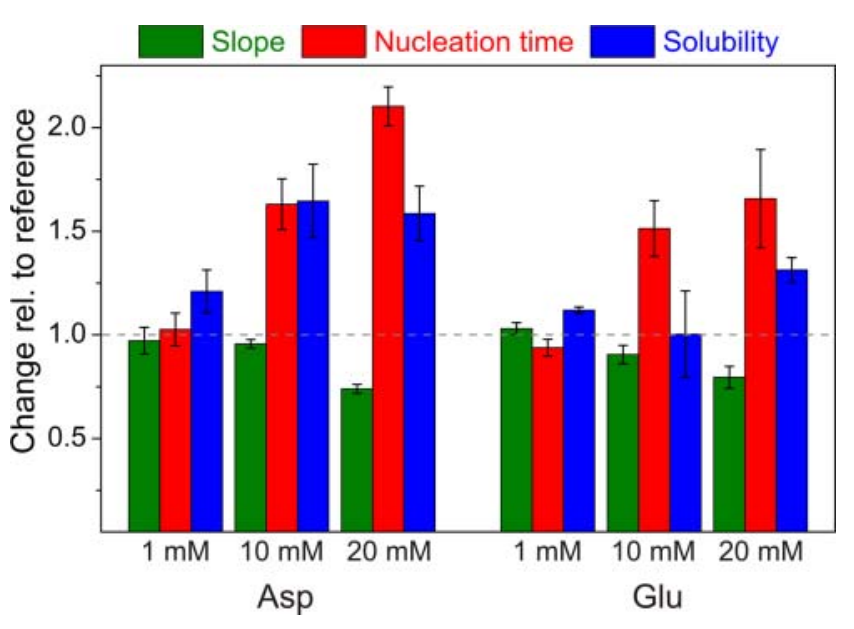

Fig. 6. Influence of the stereochemical configuration of Asp and Glu on the degree of their respective influence at different stages of $\mathrm{CaCO}_{3}$ precipitation ( $\mathrm{pH} 9.75,10 \mathrm{mM}$ amino acid in all cases).

tion of $10 \mathrm{mM}$ and a $\mathrm{pH}$ of 9.75). Comparative bar plots illustrating the observed effects are shown in Fig. 6.

Differences in the prenucleation slope of the titration curves are generally rather small and well within the limits of error. However, while average slope values determined for the L- and D-forms almost coincide $\left(4.91 \cdot 10^{-10} \mathrm{~mol} / \mathrm{s}\right.$ for L-Asp vs. $4.90 \cdot 10^{-10} \mathrm{~mol} / \mathrm{s}$ for D-Asp, and $4.65 \cdot 10^{-10} \mathrm{~mol} / \mathrm{s}$ for L-Glu vs. $4.64 \cdot 10^{-10} \mathrm{~mol} / \mathrm{s}$ for DGlu), the increase of free $\mathrm{Ca}^{2+}$ in the presence of DL-amino acids is somewhat flatter in both cases $\left(4.65 \cdot 10^{-10} \mathrm{~mol} / \mathrm{s}\right.$ for DL-Asp and $4.34 \cdot 10^{-10} \mathrm{~mol} / \mathrm{s}$ for DL-Glu). This suggests that racemic mixtures are slightly more efficient in stabilizing prenucleation clusters than optically pure solutions.

Regarding their capability of inhibiting nucleation, aspartate enantiomers do not differ to a large degree, even though there may be a relative order of increasing delay according to DL-Asp $>$ D-Asp $>$ L-Asp (effects again being within experimental error). In the case of glutamate, nucleation times found for the L- and DL-forms agree well (5760 and $5700 \mathrm{~s}$, respectively), whereas the D-enantiomer indeed appears to be a markedly weaker inhibitor (4630 s). This might indicate that L-Glu is more efficient inhibitor of $\mathrm{CaCO}_{3}$ nucleation than its D-counterpart while, tentatively, the data support an opposite scenario for Asp. Although an explanation for the molecular basis of any such trends cannot be given at the moment, these observations again support the notion that stereochemical configurations are important when it comes to the influence of amino acids on calcium carbonate crystallization.

Further interesting effects can be observed with respect to the solubility of solid phases nucleated under the influence of distinct Asp and Glu enantiomers. On one hand, we find that both D- and DL-Glu seem to induce the formation of slightly more soluble particles $\left(K_{\mathrm{sp}} \approx 4.4 \cdot 10^{-8}\right.$ and $4.2 \cdot 10^{-8} \mathrm{M}^{2}$, as compared to a value of $3.6 \cdot 10^{-8} \mathrm{M}^{2}$ obtained for the pv-ACC precipitated from solutions containing L-Glu), suggesting that a certain amount of the Denantiomer is required for the stabilization of the more metastable phase. On the other hand, DL-Asp shows very special features that differentiate it from the optically pure forms, despite the fact the mean solubilities determined for

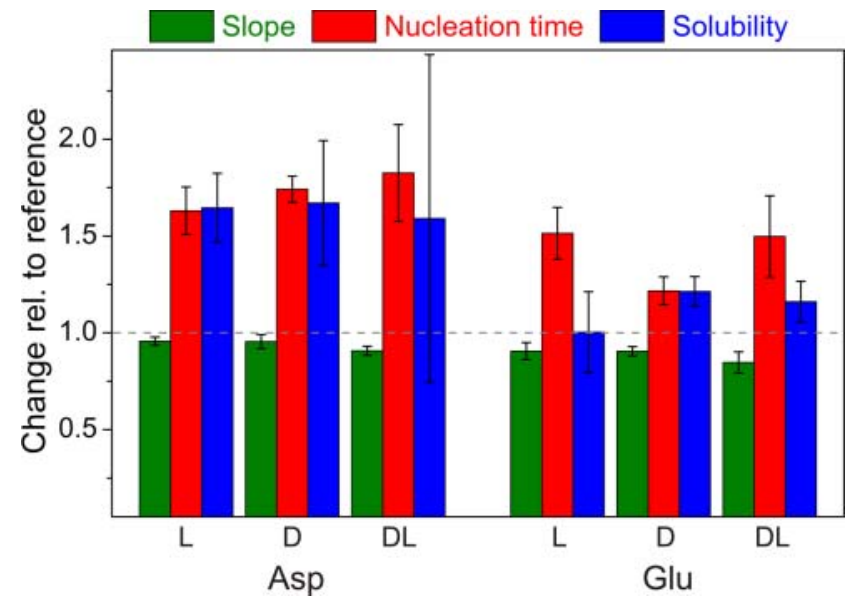

Fig. 7. Development of the free ion product in carbonate buffers containing $10 \mathrm{mM} \mathrm{DL}-\mathrm{Asp}$ at $\mathrm{pH} 9.75$, as compared to the reference experiment without additives. Note that three out of four curves (blue lines) run through a plateau at around $8-9 \cdot 10^{-8} \mathrm{M}^{2}$ before approaching the solubility product found for the reference (indicating temporary stabilization of an intermediate phase), whereas the remaining two curves (red lines) directly drop to this final level.

the three samples are nearly identical (cf. Fig. 6). A series of titration curves recorded in the presence of DL-Asp is reproduced in Fig. 7.

It is evident that, for two out of five measurements (red curves in Fig. 7), the free ion product drops directly to about the same level as in the reference, whereas there is an intermediate plateau at around $9 \cdot 10^{-8} \mathrm{M}^{2}$ in the other three cases (blue curves in Fig. 7, one of which nicely illustrating the transition from the plateau to the final level during the later stages). Thus, DL-Asp is able to temporarily stabilize a phase that is significantly more soluble than those nucleated under the influence of both $\mathrm{L}$ - and D-Asp $\left(K_{\mathrm{sp}} \approx 5.9 \cdot 10^{-8}\right.$ and $6.0 \cdot 10^{-8} \mathrm{M}^{2}$, respectively). Moreover, while the latter phases persisted in solution over the entire duration of the experiments (see Fig. 2d), particles precipitated initially in the presence of the racemic mixture were rather short-lived and transformed fairly soon - or did not occur in measureable amounts at all, as for the red curves in Fig. 7. We speculate that this intermediate phase relates to a liquid-like (PILP) precursor [58-60], which is transient to a degree that it becomes a matter of statistics whether it can be detected or not (leading to the extremely large error bars of the average solubility value shown in Fig. 6). Indeed the higher solubility observed in presence of $\operatorname{Asp}\left(K_{\mathrm{sp}} \approx 9 \cdot 10^{-8} \mathrm{M}^{2}\right)$ is still significantly lower than that found for polyaspartic acid $\left(K_{\mathrm{sp}} \approx 2 \cdot 10^{-7} \mathrm{M}^{2}\right)$ [46], but supports the possibility that already monomeric aspartate can induce a PILP precursor.

The present data suggest that the lifetime and structural nature (as reflected by the solubility) of these precursor species intimately depend on the stereochemistry of the amino acid additives, with optically pure systems yielding slightly more stable and persistent phases. Possibly, this behavior originates from distinct tendencies of the enantiomers to associate into supramolecular assemblies [49], which may be the actually relevant species for interaction with calcium carbonate. Finally, it is worth noting that the solubility of the more stable phase traced in the presence 
of DL-Asp (red curves in Fig. 7) is around $3.0 \cdot 10^{-8} \mathrm{M}^{2}$ and thus corresponds to pc-ACC, rather than pv-ACC as would be expected for the given $\mathrm{pH}$ (and observed in the reference experiment). This further corroborates the above notion that aspartate favors interaction with proto-calcitic precursors and induces nucleation of pc-ACC.

In summary, these results illustrate that amino acid chirality can indeed have a significant bearing on the influence of the molecules as additives during $\mathrm{CaCO}_{3}$ mineralization. This is essentially in line with previous studies [38], even though the detailed physical-chemical origins of corresponding effects remain as yet uncertain.

\section{Gas diffusion experiments}

In addition to the titration measurements discussed in the previous sections, the effect of amino acids on the crystallization of calcium carbonate was studied in a series of gas diffusion experiments, so as to possibly identify correlations between the interactions observed during the early stages of precipitation and changes in morphology and/or polymorphism of late (ripened) $\mathrm{CaCO}_{3}$ crystals. To that end, all $20 \mathrm{~L}$-amino acids were investigated in a conventional setup under identical conditions $(10 \mathrm{mM}$ amino acid ( $1 \mathrm{mM}$ for Tyr) in $10 \mathrm{mM} \mathrm{CaCl} 2$, exposed to ammonium carbonate vapor in a closed desiccator), and crystals formed after two weeks were characterized with respect to size, shape, and crystal phase.

Analyses of the data show that, in fact, most of the amino acids do not affect the output of crystallization to any significant extent, yielding crystals that are very similar to those obtained from reference experiments without additives (Fig. 8a) even though it is known that they become incorporated into the calcite lattice [40]. The only clear influence could be distinguished for aspartate (Fig. 8b-d), which also showed the most pronounced effects in the titration measurements.

In principle, there appear to be three distinct modes of action. First, the presence of L-Asp results in much smaller crystals $(20-40 \mu \mathrm{m}, c f$. Fig. 8 b), as compared to the typical size of the calcite rhombohedra formed in the con- trol experiment (ca. $100 \mu \mathrm{m}, c f$. Fig. 8a). This drastic decrease in crystal size might be due to higher levels of supersaturation at the point of nucleation, leading to a larger number of nuclei that on average grow to smaller dimensions. Such a scenario would generally be consistent with the titration data ( $c f$. Fig. 2d), but differences in the effective supersaturation at nucleation in the presence and absence of Asp are actually too small to account for the observed dramatic changes in size. Alternatively, aspartate could reduce the size of the crystals by adsorbing on their surface and inhibiting growth, thereby keeping primary particles small. However, recent modeling results indicate that adsorption of Asp on flat $\{104\}$ faces of calcite is rather unfavorable [49] and more likely to occur on growth kinks or edges (as reported for L- and D-Asp in earlier work [37]), or possibly other, less commonly exposed planes (see below).

The second effect of Asp refers to the polymorphic outcome of the gas diffusion experiments. While all three anhydrous crystalline modifications could be detected in the reference samples, there was strong preference for calcite discernible when Asp was present (as judged from the morphologies seen in optical micrographs (Fig. 8b), and confirmed by X-ray diffraction). This is well in line with results of the titration measurements, which indicate that Asp preferentially interacts with proto-calcitic precursors and favors precipitation of pc-ACC. Thus, added Asp seems to shift polymorphic ratios for the benefit of calcite, and perhaps does so by already controlling early-stage proto-structures.

Finally, as a third effect, aspartate modulates the morphology of calcite crystals in a way that many of the observed rhombohedra do no longer exhibit sharp and defined edges, but have a rounded appearance instead (even though the characteristic overall rhombohedral shape can still be recognized, $c f$. Fig. 6c and d). Similar morphologies have previously been obtained in the presence of different polymeric additives [14, 37, 63-65] as well as negatively charged silicate species [66]. In some of these cases, truncation of edges and corners was ascribed to face-selective adsorption of the additives, which thus be-
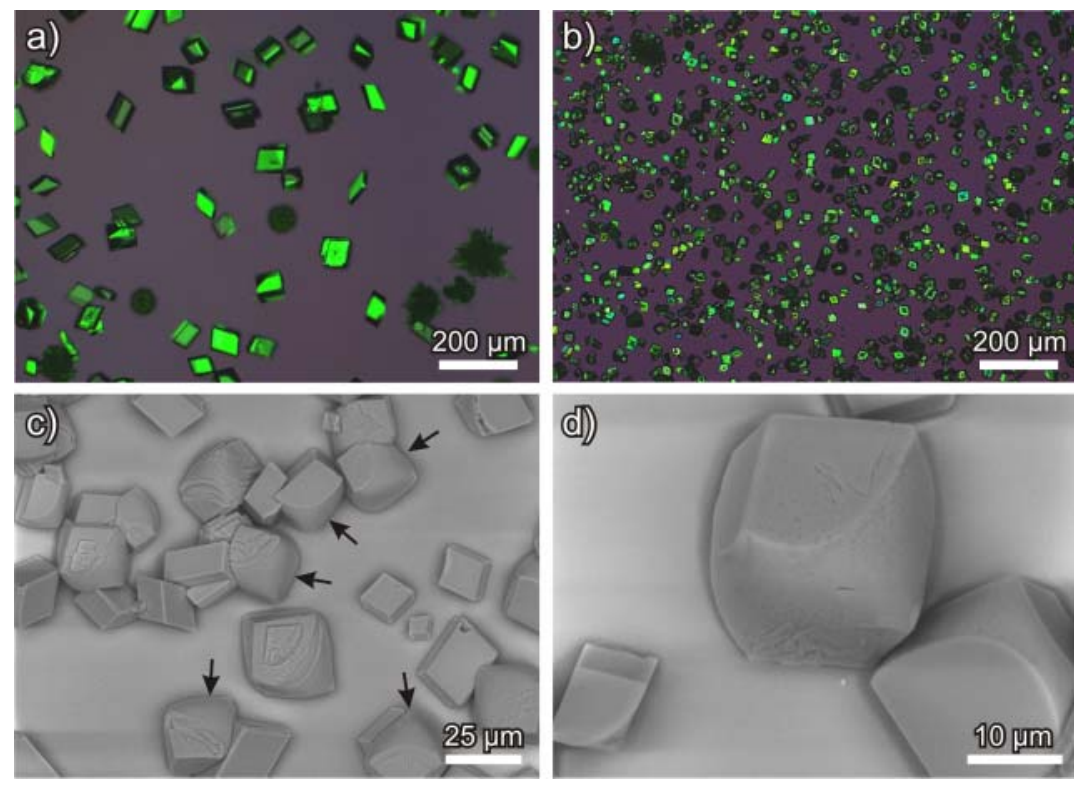

Fig. 8. Calcium carbonate crystals grown via gas diffusion without any additives (a) and in the presence of $10 \mathrm{mM} \mathrm{L-Asp} \mathrm{(b-d).} \mathrm{Polarized} \mathrm{optical} \mathrm{micro-}$ graphs show that the crystals formed under the influence of aspartic acid (b) are significantly smaller than those obtained in its absence (a), and that less vaterite and aragonite particles are found when the amino acid has been added. SEM images (c-d) disclose that calcite rhombohedra grown in the presence of L-Asp exhibit rounded edges and corners (indicated by arrows in $(\mathbf{c})$ ), which display a somewhat roughened texture. 
came stabilized and exposed. Most probably, this mechanism is also active in the present systems, possibly combined with binding of the amino acid on distinct growth steps of the calcite $\{104\}$ planes, as proposed by Orme et al. [37]. In any case, it is worth stressing that glutamate - which differs from Asp by merely one methylene group - did not cause any such effects and yielded crystals that were virtually indistinguishable from those formed in the control experiment. This is clear evidence that the influence of aspartate cannot rely on its chemical function (i.e. single negative charge) alone, but that delicate stereochemical aspects play a crucial role likewise. Indeed, similar observations have already been reported in literature. For example, Pokroy et al. found that the longer side chain of Glu hindered its incorporation into the calcite lattice [40] while Marin et. al. postulated earlier that the higher content of Asp as compared with Glu in intracrystalline proteins can be explained by differences in the chain length [11].

\section{Conclusions}

In this work, we studied the effects of the 20 natural Lamino acids on the crystallization of calcium carbonate, specifically focusing on interactions occurring in the prenucleation regime, at nucleation, during the early postnucleation stage, as well as in the course of crystal growth. Utilizing potentiometric titration measurements, the effects of additives were quantified in terms of a) their influence on the prenucleation cluster equilibrium, b) their capability to inhibit nucleation, and c) their impact on the solubility (and hence nature) of the initially precipitated solid phase. Any possible interference during growth was assessed independently by means of gas diffusion crystallization experiments.

The only amino acid found to be active in all of the investigated stages was aspartate. In addition to stabilizing prenucleation clusters and delaying nucleation as compared to reference samples, Asp induced the formation of a more soluble (i.e. less stable) initial phase and favored growth of calcite over other crystalline polymorphs. Final crystals obtained under these conditions were moreover smaller than those observed in control experiments, and had a rounded appearance due to truncation of edges and corners, possibly as a consequence of selective adsorption phenomena. This is of particular interest when considering that none of the other amino acids did modulate the morphology (and polymorphism) of $\mathrm{CaCO}_{3}$ crystals to any significant extent in this study. Regarding the interaction of the additives with prenucleation clusters, we found that - apart from Asp - also glutamate and the OH-bearing amino acids serine and threonine (and in part tyrosine as well) lead to an increased stability of the clusters, whereas alanine had a slight opposite effect. Inhibition of nucleation was observed for many of the amino acids although, again, Asp and Glu as well as Ser turned out to be most efficient in this context. Surprisingly, the only amino acid influencing the solubility of the nucleated phase (except for Asp) was glycine, perhaps owing to its relatively small size that may allow incorporation into the precipitated particles. Concentration variations performed for Asp and Glu indicated the existence of a threshold in amino acid content (between 1 and $10 \mathrm{mM}$ in the present experiments), beyond which effects become noticeable, and moreover showed that Glu can stabilize transient phases after nucleation at sufficiently high concentrations.

Our findings show that the side chain functionality of amino acids can be crucial for interaction with crystallizing calcium carbonate. Essentially, this applies for the prenucleation stage, where acidic or hydroxyl groups seem to be required for distinct cluster stabilization. Less chemical selectivity appears to underlie the capability of inhibiting nucleation, even though certain (polar) groups $\left(-\mathrm{COOH},-\mathrm{OH},-\mathrm{SR},-\mathrm{CONH}_{2}\right)$ are evidently more efficient than others. No correlation between functionality and effect can in turn be noted for the influence of the additives on the structure and solubility of the nucleated phase. An interesting observation is that basic amino acids do hardly have any impact in either of the investigated stages. This, however, does not necessarily exclude any relevance of basic side chains in oligomeric peptides, polymers, or proteins proven to be active in $\mathrm{CaCO}_{3}$ mineralization [67].

On the other hand, the results of this work have also shown that chemical functionality alone cannot account for the whole variety of effects observed. A prominent example supporting this notion is the quite distinct behavior delineated for Glu and Asp in certain respects of crystallization. Likewise, trends discerned for these amino acids by varying the $\mathrm{pH}$ of the buffer or the chirality of the monomers suggest subtle differences in their mode of interaction with both prenucleation clusters and nucleated particles, which cannot be explained based on their chemistry alone. A possible hint in rationalizing some of our findings is the recently introduced idea of proto-structuring in $\mathrm{CaCO}_{3}$ clusters and early amorphous particles [44, 50], which might be the basis for structural differentiation. Further, one may envisage that supramolecular assemblies of amino acids, discovered recently in solutions under conditions close to those of the present work [55], could also play an important role in the interaction with precipitating calcium carbonate, and that stereochemical configuration of the amino acid monomers would be essential for any such association as well.

Finally, we regard this broad study as a first of many steps towards a better - and systematic - understanding of crystallization control by amino acid-based additives. Our results suggest that in covalent peptide oligomers and polymers (proteins), secondary and tertiary structural features should be at least as important as the primary structure. As a logical next step, future work devoted to investigating artificial short-chain peptides [68] consisting of different amino acid monomers by a methodology described here will shed light on the cooperativity of distinct functional groups. We furthermore hope that our results, in particular regarding the most efficient amino acids identified, will help in the quest for, and design of, novel crystallization additives.

Acknowledgements. M. K. is grateful to BASF SE for funding a postdoc position. 


\section{References}

[1] A. Berman, L. Addadi, A. Kvick, L. Leiserowitz, M. Nelson, S. Weiner, Intercalation of Sea Urchin Proteins in Calcite: Study of a Crystalline Composite Material. Science 1990, 250, 664667.

[2] S. Weiner, L. Addadi, Design strategies in mineralized biological materials. J. Mater. Chem. 1997, 7, 689-702.

[3] S. Weiner, H. D. Wagner, The Material Bone: Structure-Mechanical Function Relations. Annu. Rev. Mater. Sci. 1998, 28, 271-298.

[4] F. H. Wilt, Matrix and Mineral in the Sea Urchin Larval Skeleton. J. Struct. Biol. 1999, 126, 216-226.

[5] J. C. Weaver, J. Aizenberg, G. E. Fantner, D. Kisailus, A. Woesz, P. Allen, K. Fields, M. J. Porter, F. W. Zok, P. K. Hansma, P. Fratzl, D. E. Morse, Hierarchical Assembly of the Siliceous Skeletal Lattice of the Hexactinellid Sponge Euplectella aspergillum. J. Struct. Biol. 2006, $158,93-\overline{106 .}$

[6] L. Addadi, D. Joester, F. Nudelman, S. Weiner, Mollusk Shell Formation: A Source of New Concepts for Understanding Biomineralization Processes. Chem. Eur. J. 2006, 12, 980-987.

[7] H. Ehrlich, Chitin and collagen as universal and alternative templates in biomineralization. Int. Geol. $\overline{R e} \overline{v .2010}, \overline{52,661} \overline{-6} \overline{99}$.

[8] H. Ehrlich, T. Hanke, A. Frolov, T. Langrock, R. Hoffmann, C. Fischer, U. Schwarzenbolz, T. Henle, R. Born, H. Worch, Modification of collagen in vitro with respect to formation of N?-carboxymethyllysine. Int. J. Biol. Macromol. $\overline{\mathbf{2 0 0 9}} \overline{\text {, 44, 51 }}$-5 6 .

[9] H. Ehrlich, T. Hanke, P. Simon, R. Born, C. Fischer, A. Frolov, T. Langrock, R. Hoffmann, U. Schwarzenbolz, T. Henle, V. V. Bazhenov, H. Worch, Carboxymethylation of the fibrillar collagen with respect to formation of hydroxyapatite. J. Biomed. Mater. Res. 2010, 92B, 542-551.

[10] B.-A. Gotliv, N. Kessler, J. L. Sumerel, D. E. Morse, N. Tuross, L. Addadi, S. Weiner, Asprich: A Novel Aspartic Acid-Rich Protein Family from the Prismatic Shell Matrix of the Bivalve Atrina rigida. ChemBioChem 2005, 6, 304-314.

[11] F. Marin, G. Luquet, B. Marie, D. Medakovic, Molluscan Shell Proteins: Primary Structure, Origin, and Evolution. In Current Topics in Developmental Biology; G. P. Schatten (ed.), Elsevier, 2007, Vol. 80, pp. 209-276.

[12] D. A. Carrino, J. P. Rodriguez, A. I. Caplan, Dermatan Sulfate Proteoglycans from the Mineralized Matrix of the Avian Eggshell. Connect. Tissue Res. 1997, 36, 175-193.

[13] M. S. Fernandez, M. Araya, J. L. Arias, Eggshells are shaped by a precise spatio-temporal arrangement of sequentially deposited macromolecules. Matrix Biol. 1997, 16, 13-20.

[14] S. Albeck, S. Weiner, L. Addadi, Polysaccharides of Intracrystalline Glycoproteins Modulate Calcite Crystal Growth In Vitro. Chem. Eur. J. 1996, 2, 278-284.

[15] S. Weiner, L. Hood, Soluble-protein of organic matrix of mollusk shells - Potential template for shell formation. Science 1975, 190, 987-988.

[16] M. Suzuki, K. Saruwatari, T. Kogure, Y. Yamamoto, T. Nishimura, T. Kato, H. Nagasawa, An Acidic Matrix Protein, Pif, is a Key Macromolecule for Nacre Formation. Science 2009, 325, $1388-1390$.

[17] C. Killian, F. H. Wilt, Characterization of the Proteins Comprising the Integral Matrix of Strongylocentrotus purpuratus embryonic spicules. J. Biol. Chem. 1996, 271, 9150-9159.

[18] S. Collino, J. S. Evans, Molecular specifications of a mineral modulation sequence derived from the aragonite-promoting protein n16. Biomacromolecules 2008, 9, 1909-1918.

[19] W. Jahnen-Dechent, C. Schäfer, M. Ketteler, M. McKee, Mineral chaperones: a role for fetuin-A and osteopontin in the inhibition and regression of pathologic calcification. J. Mol. Med. 2008, 86, 379-89.

[20] W. Jahnen-Dechent, A. Heiss, C. Schäfer, M. Ketteler, Fetuin-A regulation of calcified matrix metabolism. Circ. Res. 2011, 108, 1494-1509.

[21] M. Peled-Kamar, P. Hamilton, F. H. Wilt, Spicule Matrix Protein LSM34 Is Essential for Biomineralization of the Sea Urchin Spicule. Exp. Cell Res. 2002, 272, 56-61.

[22] M. Sarikaya, C. Tamerler, A. K. Y. Jen, K. Schulten, F. Baneyx, Molecular biomimetics: nanotechnology through biology. Nat. Mater. 2003, 2, 577-585.
[23] M. Michenfelder, G. Fu, C. Lawrence, J. C. Weaver, B. A. Wustman, L. Taranto, J. S. Evans, Characterization of two molluscan crystal-modulating biomineralization proteins and identification of putative mineral binding domains. Biopolymers 2003, 70, 522-533.

[24] M. Ndao, E. Keene, F. F. Amos, G. Rewari, C. B. Ponce, L. Estroff, J. S. Evans, Intrinsically Disordered Mollusk Shell Prismatic Protein That Modulates Calcium Carbonate Crystal Growth. Biomacromolecules 2010, 11, 2539-2544.

[25] V. N. Uversky, Natively unfolded proteins: A point where biology waits for physics. Protein Sci. 2002, 11, 739-756.

[26] F. F. Amos, J. S. Evans, AP7, a partially disordered pseudo CRING protein, is capable of forming stabilized aragonite in vitro. Biochemistry 2009, 48, 1332-1339.

[27] E. Keene, J. S. Evans, L. Estroff, Matrix-Interactions in Biomineralization: Aragonite nucleation by an intrinsically disordered nacre polypeptide, $\mathrm{n} 16 \mathrm{~N}$, associated with a beta-chitin substrate. Cryst. Growth Des. 2010, 10, 1383-1389.

[28] B. A. Wustman, D. E. Morse, J. S. Pedersen, Structural Characterization of the N-Terminal Mineral Modification Domains from the Molluscan Crystal-Modulating Biomineralization Proteins, AP27 and AP24. Biopolymers 2004, 74, 363-376.

[29] G. Z. Xu, J. S. Evans, Model peptide studies of sequence repeats from the intracrystalline biomineralization protein, SM50. I. GVGGR and GMGGQ repeats. Biopolymers 1999, 49, 303-312.

[30] F. F. Amos, M. Ndao, C. B. Ponce, J. S. Evans, A C-RING-like domain participates in protein self-assembly and mineral nucleation. Biochemistry 2011, 50, 8880-8887.

[31] B. Zhang, G. Z. Xu, J. S. Evans, Model peptide studies of sequence repeats derived from the intracrystalline biomineralization protein, SM50. II. Pro,Asn-Rich tandem repeats. Biopolymers 2000, 54, 464-475.

[32] R. A. Metzler, G. A. Tribello, M. Parrinello, P. U. P. A. Gilbert, Asprich peptides are occluded in calcite and permanently disorder biomineral crystals. J. Am. Chem. Soc. 2010, 132, 11585-11591.

[33] R. R. Naik, S. J. Stringer, G. Agarwal, S. E. Jones, M. O. Stone, Biomimetic synthesis and patterning of silver nanoparticles. Nat. Mater. 2002, 1, 169-172.

[34] R. A. Metzler, J. S. Evans, C. E. Killian, D. Zhou, T. H. Churchill, N. P. Appathurai, S. N. Coppersmith, P. U. P. A. Gilbert, Nacre Protein Fragment Templates Lamellar Aragonite Growth. $\overline{J . A m}$. $\overline{\text { Chem }} . \overline{\text { Soc. 2010, }} \overline{132,6329-6334}$.

[35] S. R. Whaley, D. S. English, E. L. Hu, P. F. Barbara, A. M. Belcher, Selection of peptides with semiconductor binding specificity for directed nanocrystal assembly. Nature 2000, 405, $665-668$.

[36] S. W. Lee, S. K. Lee, A. M. Belcher, Virus-Based Alignment of Inorganic, Organic, and Biological Nanosized Materials. $A d v$. Mater. 2003, 15, 689-692.

[37] C. A. Orme, A. Noy, A. Wierzbicki, M. T. McBride, M. Grantham, H. H. Teng, P. M. Dove, J. J. DeYoreo, Formation of chiral morphologies through selective binding of amino acids to calcite surface steps. Nature 2001, 411, 775-779.

[38] S. E. Wolf, N. Loges, B. Mathiasch, M. Panthöfer, I. Mey, A. Janshoff, W. Tremel, Phase Selection of Calcium Carbonate through the Chirality of Adsorbed Amino Acids. Angew. Chem. Int. Ed. 2007, 46, 5618-5623.

[39] A.-J. Xie, Y.-H. Shen, C.-Y. Zhang, Z.-W. Yuan, X.-M. Zhu, Y.M. Yang, Crystal growth of calcium carbonate with various morphologies in different amino acid systems. J. Cryst. Growth 2005, 285, 436-443.

[40] S. Borukhin, L. Bloch, T. Radlauer, A. H. Hill, A. N. Fitch, B. Pokroy, Screening the Incorporation of Amino Acids into an Inorganic Crystalline Host: the Case of Calcite. Adv. Funct. Mater. 2012, DOI: 10.1002/adfm.201201079.

[41] L. Addadi, J. Moradian, E. Shay, N. G. Maroudas, S. Weiner, A Chemical Model for the Cooperation of Sulfates and Carboxylates in Calcite Crystal Nucleation: Relevance to Biomineralization. Proc. Natl. Acad. Sci. USA 1987, 84, 2732-2736.

[42] N. Gehrke, H. Cölfen, N. Pinna, M. Antonietti, N. Nassif, Superstructures of Calcium Carbonate Crystals by Oriented Attachment. Cryst. Growth Des. 2005, 5, 1317-1319.

[43] A. C. Neira, M. S. Fernández, J. Reutert, Effect of the crystallization chamber design on the polymorphs of calcium carbonate 
using the sitting-drop method. In Architecture and application of biomaterials and biomolecular materials; J. Y. Wong (ed.), Materials Research Society: Warrendale, Pa., 2004.

[44] D. Gebauer, A. Völkel, H. Cölfen, Stable prenucleation calcium carbonate clusters. Science 2008, 322, 1819-1822.

[45] D. Gebauer, H. Cölfen, A. Verch, M. Antonietti, The multiple roles of additives in $\mathrm{CaCO}_{3}$ crystallization: A quantitative case study. Adv. Mater. 2009, 21, 435-439.

[46] A. Verch, D. Gebauer, M. Antonietti, H. Cölfen, How to control the scaling of CaCO3: A "fingerprinting technique" to classify additives. Phys. Chem. Chem. Phys. 2011, 13, 16811-16820.

[47] D. Gebauer, A. Verch, H. G. Börner, H. Cölfen, Influence of selected artificial peptides on calcium carbonate precipitation a quantitative study. Cryst. Growth Des. 2009, 9, 2398-2403.

[48] R. Demichelis, P. Raiteri, J. D. Gale, D. Quigley, D. Gebauer, Stable prenucleation mineral clusters are liquid-like ionic polymers. Nat. Commun. $\overline{\mathbf{2 0 1 1}, 2}, \overline{590 .}$

[49] P. Raiteri, R. Demichelis, J. D. Gale, M. Kellermeier, D. Gebauer, D. Quigley, L. B. Wright, T. R. Walsh, Exploring the influence of organic species on pre- and post-nucleation calcium carbonate. Faraday Discuss. 2012, DOI: 10.1039/c2fd20052j.

[50] D. Gebauer, P. N. Gunawidjaja, J. Y. P. Ko, Z. Bacsik, B. Aziz, L. J. Liu, Y. F. Hu, L. Bergström, C. W. Tai, T. K. Sham, M. Edén, N. Hedin, Proto-calcite and proto-vaterite in amorphous calcium carbonates. Angew. Chem. Int. Ed. 2010, 49, 8889-8891.

[51] A. R. Finney, P. M. Rodger, Probing the structure and stability of calcium carbonate pre-nucleation clusters. Faraday Discuss. 2012, DOI: $10.1039 / \mathrm{c} 2 \mathrm{fd} 20054 \mathrm{f}$.

[52] D. Hasson, H. Shemer, A. Sher, State of the Art of Friendly "Green" Scale Control Inhibitors: A Review Article. Ind. Eng. Chem. Res. 2011, 50, 7601-7607.

[53] B. Njegić-Džakula, G. Falini, L. Brečević, Ž. Skoko, D. Kralj, Effects of initial supersaturation on spontaneous precipitation of calcium carbonate in the presence of charged poly-1-amino acids. J. Colloid Interface Sci. 2010, 343, 553-563.

[54] M. Kellermeier, D. Gebauer, E. Melero-García, M. Drechsler, Y. Talmon, L. Kienle, H. Cölfen, J. M. García-Ruiz, W. Kunz, Colloidal stabilization of calcium carbonate prenucleation clusters with silica. Adv. Funct. Mater. 2012, DOI: 10.1002/ adfm.201200953.

[55] M. Kellermeier, R. Rosenberg, A. Moise, U. Anders, M. Przybylski, H. Cölfen, Amino acids form prenucleation clusters: ESI-MS as a fast detection method in comparison to analytical ultracentrifugation. Faraday Discuss. 2012, DOI: 10.1039/ c2fd20060k.
[56] S. He, J. W. Morse, The carbonic acid system and calcite solubility in aqueous $\mathrm{Na}-\mathrm{K}-\mathrm{Ca}-\mathrm{Mg}-\mathrm{Cl}-\mathrm{SO}_{4}$ solutions from 0 to $90^{\circ}$ C. Geochim. Cosmochim. Acta 1993, 57, 3533-3554.

[57] D. K. Gledhill, J. W. Morse, Calcite solubility in $\mathrm{Na}-\mathrm{Ca}-\mathrm{Mg}-$ Cl brines. Chem. Geol. 2006, 233, 249-256.

[58] L. B. Gower, D. J. Odom, Deposition of calcium carbonate films by a polymer-induced liquid-precursor (PILP) process. $J$. Cryst. Growth 2000, 210, 719-734.

[59] L. B. Gower, Biomimetic model systems for investigating the amorphous precursor pathway and its role in biomineralization. Chem. Rev. 2008, $108,4551-4 \overline{627}$.

[60] M. A. Bewernitz, D. Gebauer, H. Cölfen, L. B. Gower, A MetaStable Liquid Precursor Phase of Calcium Carbonate and its Interactions with Polyaspartate. Faraday Discuss. 2012, DOI: 10.1039/C2FD20080E.

[61] D. Gebauer, H. Cölfen, Prenucleation clusters and non-classical nucleation. Nano Today 2011, 6, 564-584.

[62] E. M. Pouget, P. H. H. Bomans, J. A. C. M. Goos, P. M. Frederik, G. de With, N. A. J. M. Sommerdijk, The initial stages of template-controlled $\mathrm{CaCO} 3$ formation revealed by cryo-TEM. Science 2009, 323, 1455-1458.

[63] R.-Q. Song, A.-W. Xu, M. Antonietti, H. Cölfen, Calcite Crystals with Platonic Shapes and Minimal Surfaces. Angew. Chem. Int. Ed. 2009, 48, 395-399.

[64] R.-Q. Song, H. Cölfen, A.-W. Xu, J. Hartmann, M. Antonietti, Polyelectrolyte-Directed Nanoparticle Aggregation: Systematic Morphogenesis of Calcium Carbonate by Nonclassical Crystallization. ACS Nano 2009, 3, 1966-1978.

[65] D. B. DeOliveira, R. A. Laursen, Control of Calcite Crystal Morphology by a Peptide Designed To Bind to a Specific Sur: face. J. Am. Chem. Soc. 1997, 119, 10627-10631.

[66] M. Kellermeier, E. Melero-García, W. Kunz, J. M. García-Ruiz, The Ability of Silica to Induce Biomimetic Crystallization of Calcium Carbonate. In Advances in Chemical Physics; G. Nicolis, D. Maes (eds.), John Wiley \& Sons, Inc.: Hoboken, NJ, USA, 2012; pp. 277-307.

[67] B. Cantaert, Y.-Y. Kim, H. Ludwig, F. Nudelman, N. A. J. M. Sommerdijk, F. C. Meldrum, Think Positive: Phase Separation Enables a Positively Charged Additive to Induce Dramatic Changes in Calcium Carbonate Morphology. Adv. Funct. Mater. 2012, 22, 907-915.

[68] V. Dmitrovic, G. J. M. Habraken, M.-M. R. M. Hendrix, W. J. E. M. Habraken, A. Heise, G. de With, N. A. J. M. Sommerdijk, Random Poly(Amino Acid)s Synthesized by Ring Opening Polymerization as Additives in the Biomimetic Mineralization of $\mathrm{CaCO}_{3}$. Polymers 2012, 4, 1195-1210. 